\title{
On Services and Insights of Technology Intelligence System
}

\author{
Seungwoo Lee, Minhee Cho, Sa-Kwang Song, and Hanmin Jung \\ Department of Computer Intelligence Research, Korea Institute of Science and Technology \\ Information, 245 Daehak-ro, Yuseong-gu, Daejeon, 305-806, Korea \\ \{swlee, mini, esmallj, jhm\}@kisti.re.kr
}

\begin{abstract}
The importance of technology strategy in business is getting emphasized as global technology competition is being rapidly intensified. To achieve successful business, nothing is more important than timely establishment of proper technology strategy. The process of establishing technology strategy is technology planning, which should be supported by technology intelligence (TI). To reduce the cost of manual technology intelligence activities, we suggest an automated technology intelligence system which can support whole steps of technology planning systematically. We examined what decision should be made and what information is required in each step. And then, we suggested seven services and their explicit insights which have a specific role at each step of technology planning. Considering recent growth of mobile environment of users, we implemented our system running on tablet PCs.
\end{abstract}

Keywords: technology planning, strategy planning, technology intelligence, knowledge acquisition, information analytics, insight.

\section{$1 \quad$ Introduction}

As business and technology environment rapidly changes and global technology competition is being gradually intensified, the importance of technology strategy as well as business strategy (or strategic management) is getting emphasized. Business strategy identifies the objectives of a particular organization (institution or company) and defines the plans and actions to achieve the objectives [1], while technology strategy defines the objectives, strategies and tactics related to development and application of technologies within an organization [2]. To achieve successful business, nothing is more important than timely establishment of proper technology strategy in addition to business strategy. That is, an organization should continue to discover emerging and promising technologies for its business and make a timely plan of when and how to acquire such technologies. This process of establishing technology strategy is technology planning, which should be supported by technology intelligence (TI). Technology intelligence includes a wide variety of activities such as gathering, analyzing and forwarding information on new technologies to support technology planning and decision-making of an organization [3]. 
To perform proper technology planning, each organization needs to perform active, continuous and objective technology intelligence activities. However, these activities require many human resources including domain experts and cost too much for small and medium-sized enterprises (SMEs) to come up with. Some analytics tools such as $\mathrm{SAS}^{1}$ or VantagePoint ${ }^{2}$ provide useful analytic functionalities for technology intelligence, but they require a user to learn advanced skills for correct usage of them and to prepare his or her own source data to be analyzed. Furthermore, those tools do not support the whole steps of technology planning systematically. On the other hand, existing information retrieval systems like Google Search ${ }^{3}$ (or Google Scholar Search $^{4}$ ) provide a cheap means of finding technical literature such as papers and patents, but they have a certain limit to their usefulness because they just list up too many results. So, this paper suggests an automated technology intelligence service which can be used with ease like existing information retrieval systems and can support the whole steps of technology planning systematically.

The rest of this paper is organized as follows. In Section 2, we surveys related work to analytics for technology intelligence and then we explore the steps of technology planning in Section 3. We investigate what is needed for each step of technology planning and suggest processes for knowledge acquisition in Section 4, and then define services for technology intelligence in Section 5. We finally conclude in Section 6.

\section{Related Work}

Gartner ${ }^{5}$ pronounces the list of promising technologies every year. Information analytics organizations such as Gartner usually surveys and investigates promising technologies from many domain experts across the whole world through Delphi method [4], and therefore many researchers and companies refer to and rely on their pronouncement. However, it costs quite much and there are also some criticisms that the result of survey depends on subjective opinions of experts participated in the survey.

More objective analytics may be obtained from data. There have recently been some approaches which perform and provide analytics from data, beyond a typical search paradigm. Google Scholar Search and Microsoft Academic Search ${ }^{6}$ analyze and serve researchers' specialties, research topics and research network such as coauthorship and citation relationship and paths from publication data such as papers and patents. However, they are so far focusing on network analytics of researchers and organizations, not analytics for technology planning. On the other hand, Recorded Future $^{7}$ tries to analyze and predict future events. It continually collects public Web

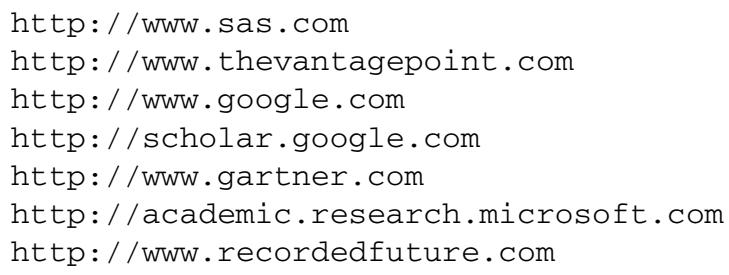


content such as online news, blogs, public niche sources, government web sites, and financial databases, etc., analyzes them to identify references to entities and events, and visualizes insights for better understanding of complex relationships and issues.

Because technology planning is an important issue for enhancing global competencies of each country, there have been performed several government-funded research projects about information analytics for business and technology intelligence. US government has supported FUSE (Foresight and Understanding from Scientific Exposition) project [5], the goal of which is to enhance inter-disciplinary, converged competitiveness by managing vast amounts and kinds of literature with a single, established system while also developing an automated method to support systematic and successive evaluation of technological potential based on information identified in the literature. EU also has supported a similar project, CUBIST (Combining and Uniting Business Intelligence with Semantic Technology) [6], which aims to develop an enhanced Semantic Web search platform to allow business-related users to better understand large and heterogeneous data. These two projects commonly have started from 2011 and combine explicit metadata used by most services with implicit metadata hidden within text documents, in order to effectively find technological potential. However, FUSE mainly focuses on quantitative analysis based on scientometrics while CUBIST mainly focuses on qualitative analysis based on Text Mining and Semantic Web technologies. Korea has also supported similar project, InSciTe (Intelligence from Science \& Technology) [7], which aims to provide technological policy makers, researchers, and small and medium-sized enterprises with technology intelligence services. InSciTe has focused on analytics of technological potential and levels of research agents. In this paper, we extend the InSciTe to support the whole steps of technology planning systematically.

\section{Technology Planning Steps}

Technology planning in an organization is generally carried out through the following five steps (see Fig.1): (1) selection and understanding of an emerging and promising technology; (2) understanding of core or strategic element technologies of the emerging and promising technology; (3) understanding of technical competitiveness and competitors; (4) establishment of technology strategy; (5) execution of technology strategy [8][9].

Among these steps, the first four steps require information to support decisionmaking by executives of an organization and such information should be acquired through technology intelligence. So, we need to examine what decision should be made and what information is required in the first four steps.

The first step should suggest an emerging and promising technology suitable for a given organization or researcher. To do this, we should also analyze the network of related technologies and evaluate the emergence of each technology and how the technology is promising. The promising degree of a technology could be computed by considering its element technologies and related products [10]. 
In the second step, core or strategic element technologies of the emerging and promising technology should be determined. To do this, we need to disassemble the selected technology in the previous step into its element technologies and should evaluate the importance of each element technology.

The third step should identify leading researchers and organizations and evaluate technological levels of them in the view of the selected technology and its element technologies.

Finally, the fourth step should determine development order and acquisition method of element technologies. To do this, we need to evaluate maturity and dependency between element technologies.

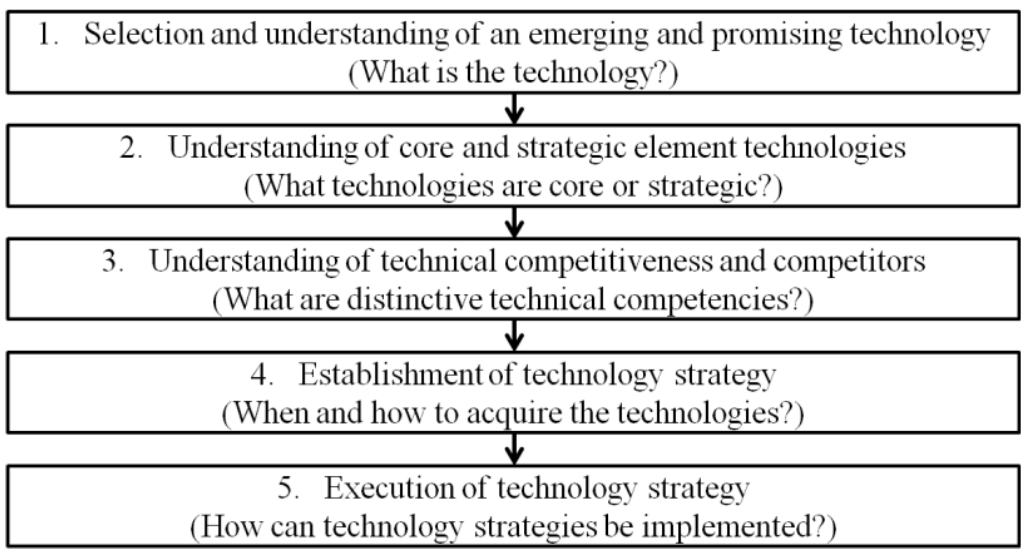

Fig. 1. Five steps of technology planning

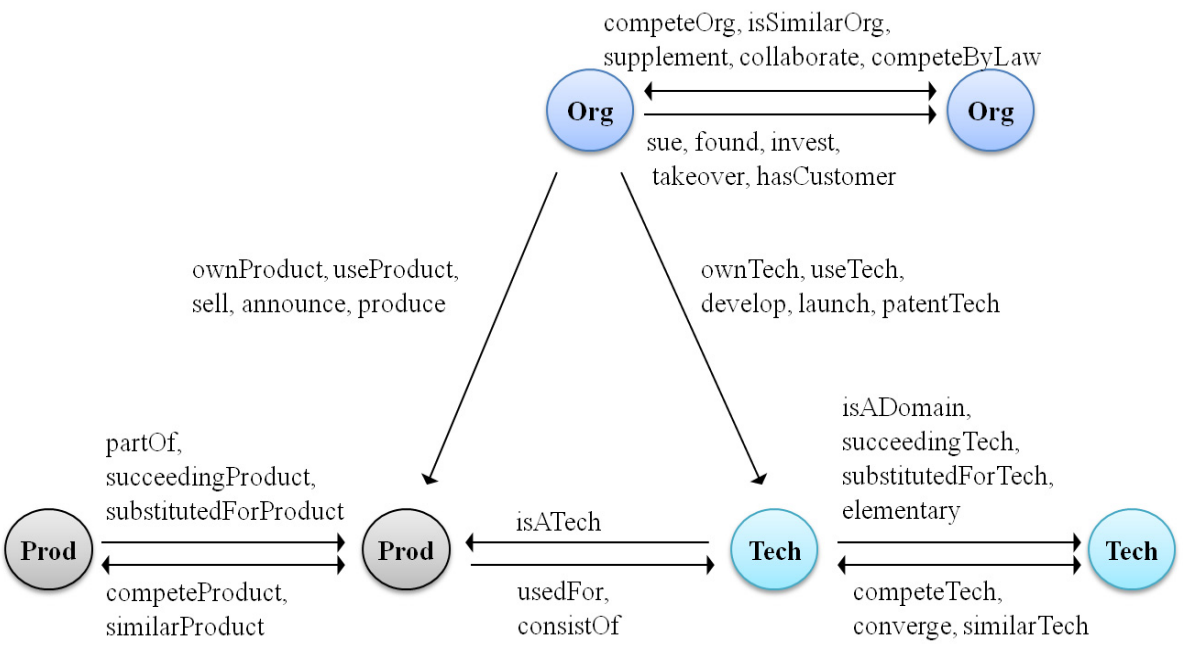

Fig. 2. Relationships defined between technology, product, and organization 


\section{$4 \quad$ Knowledge Acquisition}

From the steps of technology planning described in the previous section, we can get aware of the fact that core knowledge needed for technology planning should be composed of technology, product and organization. Here, technology includes technical areas, product types, materials, and substances, and product indicates commodities implemented by applying technologies, including intangible ones such as services [11]. Product is a very important element for technology planning because it is a mirror of technologies applied to it.

There exist various and useful relationships between these three entities for technology planning (see Fig. 2) and we can define and classify them by the technology planning steps [12]. For example, all relationships between technologies such as isADomain (is a domain of), similarTech (is a similar technology to), competeTech (is a competing technology with), and converge and some relationships between product and technology such as isATech (is a technology of; e.g., smart phone is a technology of iPhone4) and consistOf (consists of or implements; e.g., iPhone $4 S$ consists of speech recognition) could be used for technology intelligence at the first step.

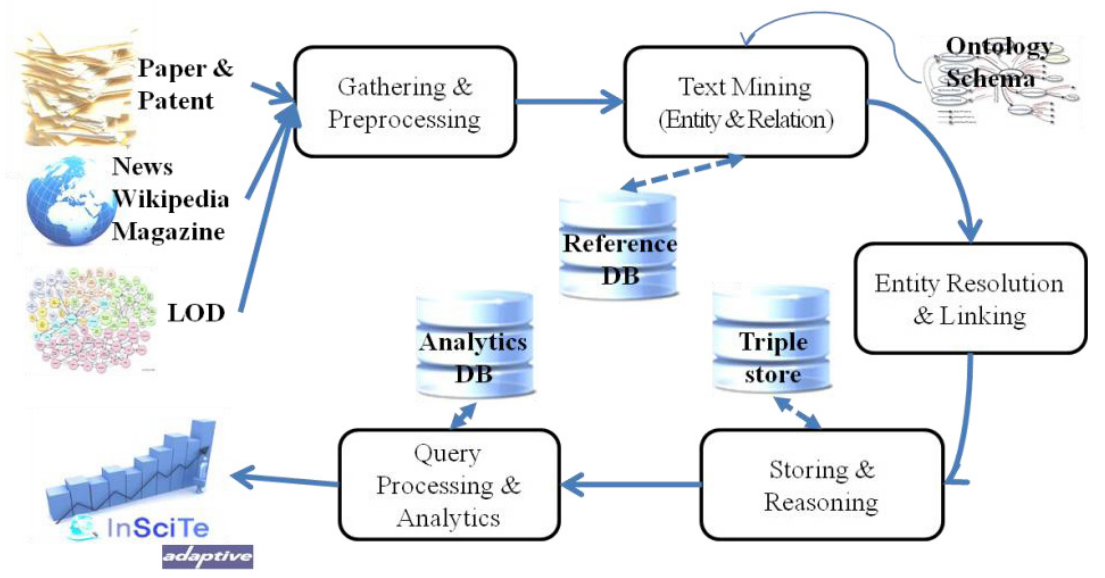

Fig. 3. Overall processes of knowledge acquisition for technology intelligence

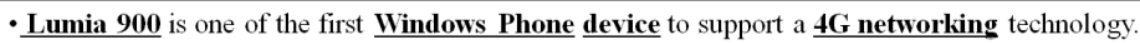

-... both Nokia and its partner Microsoft to flog the new Lumia smartphone, which runs

Windows Phone,

- ... Samsung Electronics on Thursday unveiled a new tablet PC named the Galaxy Tab ...

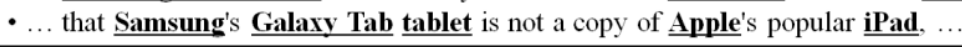

Fig. 4. The text examples excerpted from news articles

The core of technology intelligence system is to extract and make knowledge from technical literature such as papers, patents, news, magazines, and other technical Web documents in automated way. Natural language processing, text mining, and semantic 
web technology play important roles to do this. Fig. 3 shows the overall processes from gathering of technical literature to query processing. Linked Open Data (LOD) is utilized for resolving and linking same entities. Fig. 4 shows some example excerpts from news articles. Bold and underlined phrases indicate entities - technologies, products, and organizations - to be recognized and extracted. To correctly recognize these entities, we need to secure enough authority data by entity types because contexts of the entities are often not enough to recognize their types. For example, 'Windows Phone' occurs twice but its contexts are not enough to recognize that it is a product of mobile operating system unless we are aware of that in advance. Based on the extracted entities, relationships between those entities are recognized and extracted by applying pattern-based rules [13]. Fig. 5 shows the relationships extracted from the example text of Fig. 4. The double-line-boxed entities indicate product, single-line-boxed ones indicate technology, and dotted-line-boxed ones indicate organization. The extracted entities and their relations are converted into semantic triples through URI (Uniform Resource Identifier) assignment and resolution [12][14].

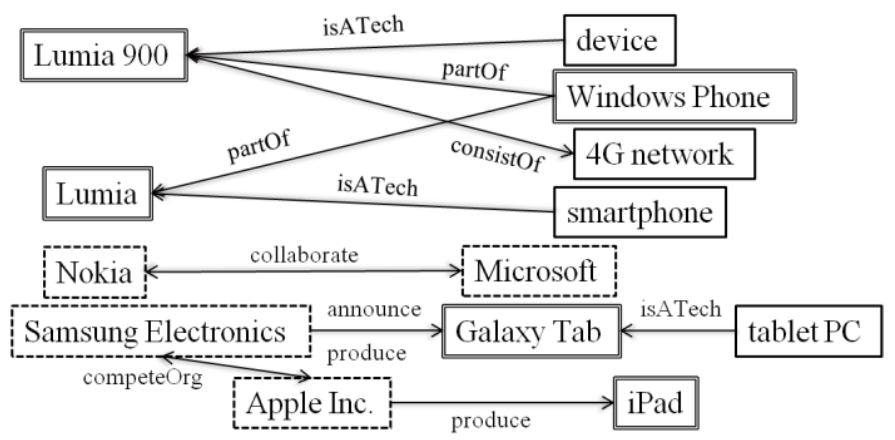

Fig. 5. The examples of entities and their relations extracted

\section{$5 \quad$ Technology Intelligence Services and Insights}

Now, we suggest which services and what insights an automated technology intelligence system should provide to support the steps of technology planning described in Section 3. Insight indicates the meaning entailed by a service result and will help users to well understand each service result.

The first step can be supported by technology navigation and technology trend services. Technology navigation provides exploration of network composed of technologies, products and organizations with relationships among them. It helps users to understand technologies by exploring the relationships. Technology trend analyzes and provides growth stage and speed of emerging technologies in technology life cycle. These services can provide users with insights such as commercialization and opportunity - promising degree - of emerging technologies.

The second step can be supported by element technology and convergence technology services. Element technology service discovers which element technologies 
compose a given technology and the growth stage and speed of each element technology. Convergence technology service identifies convergeable technologies with a given technology. These services can provide users with insights such as what is core or strategic among the element technologies and converge-ability of a given technology.

The third step can be supported by agent level and agent partner services. Agent level service compares organizations by their development levels of a given technology. It helps users to understand technological competitiveness of each organization and to identify technology competitors. Agent partner service identifies and recommends collaboration candidates among leading organizations. These services can provide users with insights such as competition or collaboration degree among organizations in the view of a given technology.

The fourth step can be supported by technology roadmap service. This service analyzes dependencies among element technologies and can provide users with insights such as which order and how to acquire each element technology.

Fig. 6 shows the technology intelligence services and their flows by the technology planning steps. After the fourth step, all the analyzed results are piled into a report to provide users.

Step1. Selection and understanding of an emerging and promising technology

Step2. Understanding of core and strategic element technologies

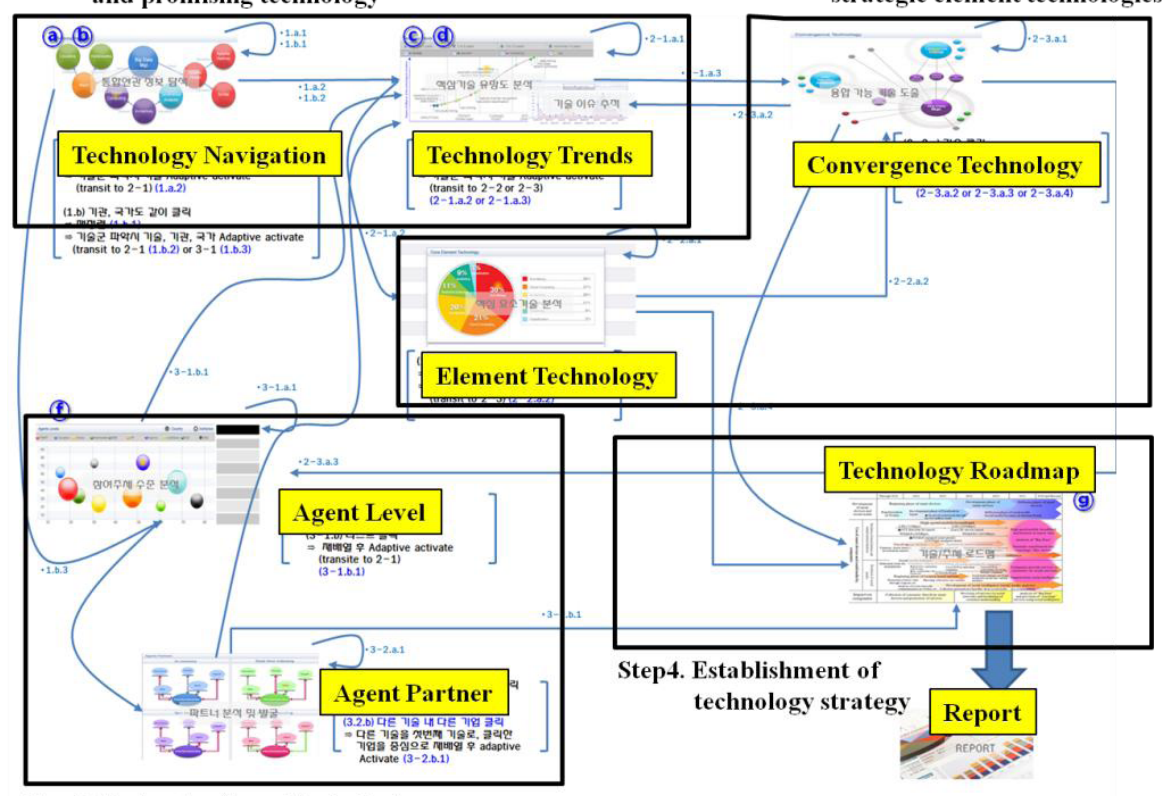

Step3. Understanding of technical competiveness and competitors

Fig. 6. Service flows by technology planning steps 


\section{Conclusion}

Technology planning is a process of establishing technology strategy and should be supported by technology intelligence (TI). To reduce the cost of manual technology intelligence activities, we suggest an automated technology intelligence system which can be used with ease like existing information retrieval systems and can support whole steps of technology planning systematically. Technology planning has five steps among which first four steps can be supported by a TI system. We examined what decision should be made and what information is required in each step. We then suggested seven services and insights which have a specific role at each step of technology planning. As the number of mobile users is rapidly growing, we implemented our system running on Android tablet PCs. In the future, we will try to compare ours to FUSE and CUBIST outcomes after they are accessible publically.

\section{References}

1. Lamb, R.B.: Competitive Strategic Management. Prentice-Hall, NJ (1984)

2. Floyd, S.W., Wolf, C.: Technology Strategy. In: Narayanan, V.K., O'Connor, G.C. (eds.) Encyclopedia of Technology and Innovation Management, pp. 125-128. John Wiley \& Sons Ltd., West Sussex (2010) ISBN-978-1-405-16049-0

3. Mortara, L., Kerr, C., Probert, D., Phaal, R.: Technology Intelligence-Identifying threats and opportunities from new technologies, University of Cambridge Institute for Manufacturing (2007) ISBN-978-1902546513

4. Linstone, H.A., Turoff, M.: The Delphi Method: Techniques and Applications. Murray Turoff and Harold A. Linstone (2002) ISBN 0-201-04294-0

5. FUSE, Foresight and Understanding from Scientific Exposition Program - Broad Agency Announcement (BAA) IARPA-BAA-10-06, http: / /www. iarpa.gov/Programs / ia/FUSE/solicitation_fuse.html (last accessed at March 1, 2013)

6. CUBIST, Combining and Uniting Business Intelligence with Semantic Technology Project, http: / /www. cubist-project.eu/ (last accessed at March 1, 2013)

7. Lee, M., Lee, S., Kim, J., Seo, D., Kim, P., Jung, H., Lee, J., Kim, T., Koo, H.K., Sung, W.K.: InSciTe Advanced: Service for Technology Opportunity Discovery. In: International Semantic Web Conference (ISWC 2011) - Semantic Web Challenge, Germany (2011)

8. Lee, J.J., Bae, J.T.: Dimension and Contents of Technology Strategy, lecture note MGT532 of KAIST (2001) (in Korean)

9. Lee, S., Cho, M., Song, S.K., Hong, S.C., Jung, H.: Strategy for Developing Technology Planning Support System. In: Proceedings of International Conferences on AST, EEC, MMHS, and AIA 2012, China, pp. 190-193 (2012)

10. Kim, J., Hwang, M., Jeong, D.H., Jung, H.: Technology Trends Analysis and Forecasting Application based on Decision Tree and Statistical Feature Analysis. Expert Systems with Applications 39, 12618-12625 (2012)

11. Cho, M., Lee, S., Song, S.K., Hong, S.C., Jung, H.: Infrastructure for supporting R\&D planning system. In: JIST 2012, Japan (2012) 
12. Lee, S., Song, S.K., Cho, M., Hong, S.C., Jung, H.: Knowledge construction strategy for technology intelligence system. In: Proceedings of the Korean Society for Internet Information Conference, Korea (2012) (in Korean)

13. Chun, H.W., Jeong, C.H., Shin, S.H., Seo, D.M., Hwang, M.N., Jang, H.J., Park, W.C., Park, J.W., Lee, S., Choi, S.P., Jung, H.: Information Extraction for Technology Trend Analysis. International Journal on Advances in Information Sciences and Service Sciences (on reviewing)

14. Kim, P., Kim, T., Lee, S., Jung, H., Sung, W.K.: OntoURIResolover: URI Resolution Service using Multiple Ontologies. In: International Semantic Web Conference (ISWC 2011) - Semantic Web Challenge, Germany (2011) 